\title{
Type 2 innate lymphoid cell counts are increased in patients with systemic sclerosis and correlate with the extent of fibrosis
}

\author{
Thomas Wohlfahrt, ${ }^{1}$ Svetlana Usherenko, ${ }^{1}$ Matthias Englbrecht, ${ }_{1}^{1}$ Clara Dees, ${ }^{1}$ \\ Stefanie Weber, ${ }^{1}$ Christian Beyer, ${ }^{1}$ Kolja Gelse, ${ }^{2}$ Oliver Distler, ${ }^{3}$ Georg Schett, ${ }^{1}$ \\ Jörg H W Distler, ${ }^{1}$ Andreas Ramming ${ }^{1}$
}

\begin{abstract}
Handling editor Tore K Kvien
- Additional material is published online only. To view please visit the journal online (http://dx.doi.org/10.1136/ annrheumdis-2015-207388)
\end{abstract}

${ }^{1}$ Department of Internal Medicine 3, Institute for Clinical Immunology, University of Erlangen-Nuremberg, Erlangen, Germany

${ }^{2}$ Department of Trauma Surgery, University of ErlangenNuremberg, Erlangen, Germany ${ }^{3}$ Division of Rheumatology, Research of Systemic Autoimmune Diseases, University Hospital Zurich, Zurich, Switzerland

\section{Correspondence to} Dr Jörg H W Distler, Department of Medicine 3, Institute for Clinical Immunology, University of Erlangen-Nuremberg, Ulmenweg 18, Erlangen D-91054, Germany; joerg.distler@uk-erlangen.de

JHWD and AR contributed equally.

Received 31 January 2015 Revised 26 June 2015 Accepted 11 August 2015 Published Online First 2 September 2015

\section{ABSTRACT}

Objective Type 2 innate lymphoid cells (ILC2s), a recently identified population of lymphoid cells lacking lineage-specific receptors, promote type 2 immunity and tissue remodelling. However, the contributive role of ILC2s in the pathogenesis of systemic sclerosis (SSc) is unknown. We aimed to evaluate the levels and correlations with fibrotic manifestations in SSc.

Methods 69 patients with SSC and 47 healthy controls were included. Blood samples and skin sections were analysed by flow cytometry and immunohistochemically by staining two complementary panels of markers.

Results Dermal and circulating ILC2s were significantly elevated in patients with SSc compared with controls. Dermal, but not circulating ILC2s were activated. Stratification of the SSC population in patients with limited cutaneous SSc (ICSSc) and diffuse cutaneous SSC (dcSSc) demonstrated increased levels of ILC2s in both subgroups with significantly higher frequencies in dcSSc compared with ICSSc. Moreover, dermal and circulating ILC2 counts correlated closely with the modified Rodnan skin score and with the presence of pulmonary fibrosis. Conclusions ILC2 counts are elevated in patients with SSc and correlate with the extent of skin fibrosis and the presence of interstitial lung disease providing compelling evidence for profibrotic effect of ILC2s in SSc.

\section{INTRODUCTION}

Tissue fibrosis affecting the skin, but also internal organs such as the lungs, is the major hallmark of systemic sclerosis (SSc). ${ }^{1}{ }^{2}$ The accumulation of extracellular matrix components disrupts the physiological tissue structure and leads to organ dysfunction, high morbidity and increased mortality of affected patients. However, the mechanisms in this fundamentally important process of tissue injury are incompletely understood. A growing body of evidence suggests that overproduction of extracellular matrix components results from complex interactions between various cells, including leucocytes and fibroblasts. ${ }^{3} 4$ More recently, locally accumulating innate-like lymphoid cells (ILCs) are emerging as an important cellular source of cytokines triggering fibrotic tissue remodelling independently of the adaptive immune system..$^{56}$

ILCs are characterised by the absence of conventional lineage markers. ${ }^{7}{ }^{8}$ Similar to $\mathrm{T}$ cells, they are categorised into three groups, according to distinct pattern of cytokine production and the requirement of specific transcription factors guiding their development and function. 910

The type 2 cytokines interleukin (IL)-25 and IL-33 are implicated in the generation of IL-13expressing type 2 innate lymphoid cells (ILC2s). In the mouse model of carbontetrachloride $\left(\mathrm{CCl}_{4}\right)$-induced liver fibrosis, ILC2s activated via ST2-dependent signalling are a major source of the profibrotic cytokine IL-13. ${ }^{5}$ Moreover, inhibition of ILC2 development dampens collagen deposition following injection of Schistosoma mansoni eggs. ${ }^{6}$ Besides the absence of conventional lineage markers, ILC2s express several characteristic receptors such as the inducible $\mathrm{T}$ cell costimulator (ICOS), the coinhibitory receptor killer-cell lectinlike receptor G1 (KLRG1) and the cytokine receptors IL-25 (IL17RB) and IL-33 (T1/ST2). ${ }^{11-15}$

Here, we identified increased frequencies of ILC2s in skin sections and in the circulation of patients with SSc compared with healthy controls (HC), suggesting that ILC2s may trigger fibroblast activation and tissue fibrosis. Furthermore, ILC2 numbers in the skin and the blood correlated with the extent of fibrosis in patients with SSc.

\section{MATERIALS AND METHODS}

Patient characteristics and materials and methods are available in the online supplementary material.

\section{RESULTS}

\section{ILC2 numbers are elevated in the skin of patients with SSC}

As no single marker can sufficiently distinguish ILC2s from other cell population, we used two established sets of ILC2 markers to quantify ILC2s in the skin of patients with SSc and healthy individuals. Using the definition of ILC2s as $\mathrm{ICOS}^{+}$; $\mathrm{ST}^{+}{ }^{+} \mathrm{CD}^{-}$; $\mathrm{CD} 11 \mathrm{~b}^{-}$cells, we observed a 10 -fold increase in ILC2s in skin sections of patients with SSc (median 4.78; IQR 2.03-11.24) compared with HC (median 0.48; IQR 0-1.91; p=0.0003) (figure 1A). Elevated ILC2 counts in the skin of patients with SSc were confirmed using an independent second panel of positive and negative markers of ILC2s. Consistent with the results obtained with the first panel of ILC2 markers, also the characterisation as ST2 ${ }^{+}$; IL- $17 \mathrm{RB}^{+}$; $\mathrm{KLRG}^{+}$; Lin $^{-}$(CD3, CD14, CD16, CD19, CD20 and 
A

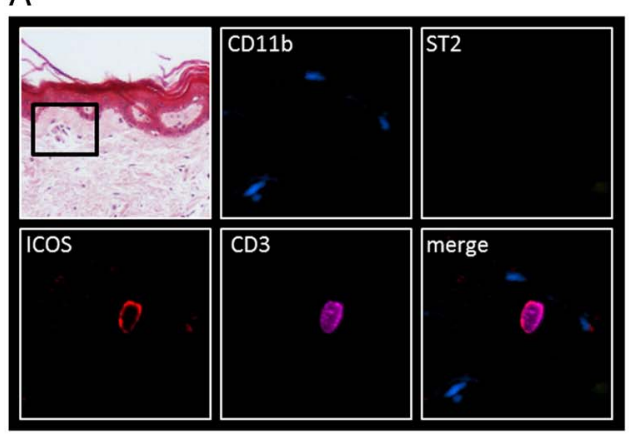

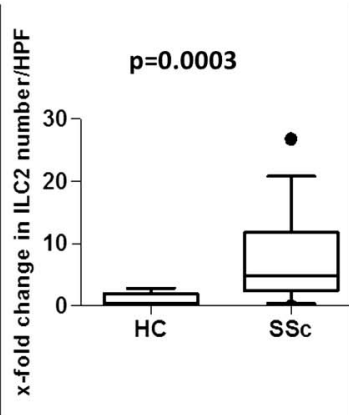

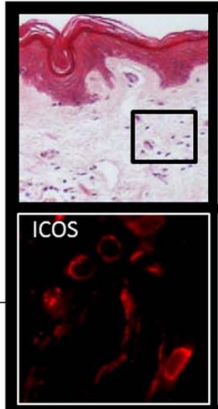

SSC

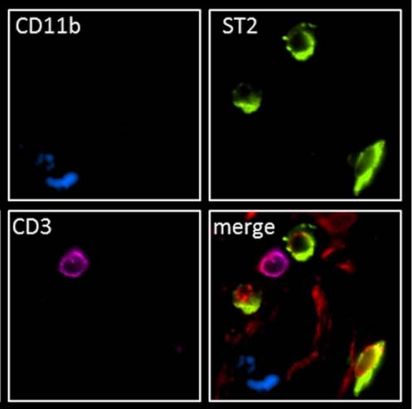

B

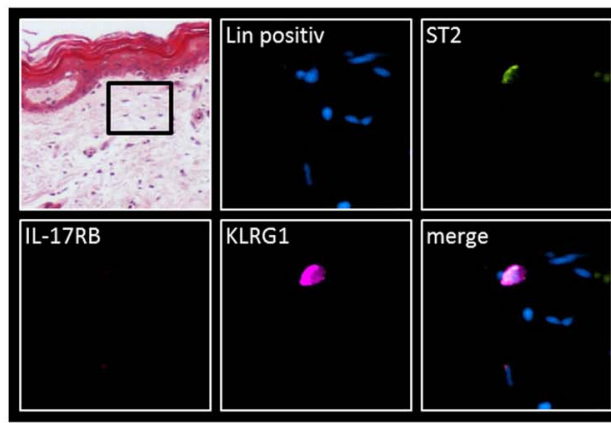

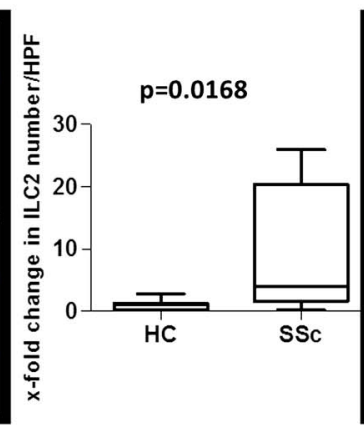

SSc

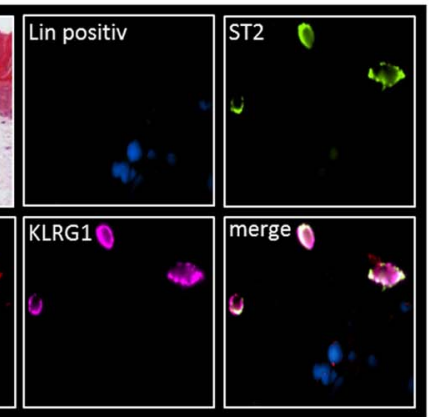

Figure 1 The number of type 2 innate lymphoid cells (ILC2s) is elevated in the skin of patients with systemic sclerosis (SSc) compared with healthy controls (HC). (A) Representative images and quantification of H\&E and immunofluorescence staining for ILC2s in the skin of patients with SSC $(n=33)$ and healthy volunteers $(n=11)$ using ST2 and ICOS as positive markers and CD11b and CD3 as negative markers for ILC2s. (B) Representative images (HC: $n=7 ;$ SSc: $n=9$ ) and quantification of H\&E and immunofluorescence staining for ILC2s using a complementary panel of markers with ST2, interleukin (IL)-17RB and killer-cell lectin-like receptor G1 (KLRG1) as positive markers and lineage markers consisting of CD3, CD14, CD16, CD19, CD20 and CD56 as negative markers. Representative images are shown at a 100-fold (H\&E) or 600-fold (immunofluorescence) magnification. All data are shown as box plots with $5 \%$ and $95 \%$ centiles. HPF, high power field.

CD56) cells showed an increase in ILC2s by 3.8 -fold in patients with SSc (median 4.03; IQR 1.91-16.12) compared with HC (median 1.06; IQR 0.32-1.27) (figure 1B). Autofluorescent mast cells and erythrocytes were excluded from analysis by toluidine-blue and nuclear (4',6-Diamidin-2-phenylindol; DAPI) staining (see online supplementary figure S1A-C).

\section{Increased ILC2 numbers in the skin of patients with SSC are associated with fibrotic manifestations}

We next analysed potential associations of ILC2 numbers with clinical manifestations in patients with SSc. We stratified the SSc population in patients with limited cutaneous SSc (lcSSc) and diffuse cutaneous SSc (dcSSc). Although the number of ILC2s in the skin was increased in both subgroups of patients with SSc compared with healthy individuals, ILC2 numbers were significantly higher in patients with dcSSc than in patients with lcSSc (figure 2A). We grouped patients with SSc into patients with restricted and extensive skin fibrosis and found that patients with a modified Rodnan skin score (mRSS) $>10$ have higher ILC2 numbers in the skin compared with patients with SSc with an mRSS $\leq 10$ (figure 2B). Moreover, dermal ILC2 counts correlated with the mRSS (figure 2C). Further characterisation of ILC2s revealed homogeneous expression of thymic stromal lymphopoietin protein (TSLP) receptor and IL-17RB in dermal, but not in circulating ILC2s, suggesting activation of ILC2s in the skin of patients with SSc (figure 2D, see online supplementary figure S4B). The majority of dermal ILC2s also express cutaneous lymphocyte antigen (CLA), suggesting putative skin homing of ILC2s in patients with SSc (see online supplementary figure S4A).

The number of circulating ILC2s is increased in patients with SSc and correlates with the extent of skin fibrosis and the presence of interstitial lung disease

As access to skin biopsies in clinical routine is limited, we analysed whether ILC2 counts may also be correlated with fibrotic manifestations in patients with SSc. Indeed, frequencies and absolute numbers of $\mathrm{CD}_{4}{ }^{+}$; $\mathrm{ST}_{2}^{+}$; $\mathrm{ICOS}^{+}$; $\mathrm{Lin}^{-}(\mathrm{CD} 3, \mathrm{CD} 14$, CD16, CD19, CD20 and CD56) ILC2s were elevated in the blood of patients with SSc compared with controls (figure 3A, see online supplementary figures S2A and S3C). Consistently, the characterisation as $\mathrm{CD} 45^{+}$; $\mathrm{CRTH} 2^{+}$; IL-7R $\alpha^{+}$; $\mathrm{Lin}^{-}(\mathrm{CD} 3$, CD14, CD16, CD19, CD20 and CD56) cells also showed significantly elevated circulating ILC2 counts in patients with SSC compared with HC (see online supplementary figures S2B and S3B, D). As for ILC2s in the skin, ILC2 numbers in the blood were higher in patients with dcSSc, and in patients with an mRSS of $>10$ compared with patients with lcSSc and mRSS of $\leq 10$, respectively (figure. $3 \mathrm{~B}, \mathrm{C}$ ). Circulating ILC2 counts also correlated closely with the mRSS $\left(\mathrm{R}^{2}=0.44 ; \mathrm{p}<0.0001\right.$; online supplementary figure S5A). Moreover, patients with SSc with pulmonary fibrosis had increased ILC2 numbers in peripheral blood compared with patients with SSc without interstitial lung disease (ILD) (figure 3D). Notably, ILC2 counts were highest in 

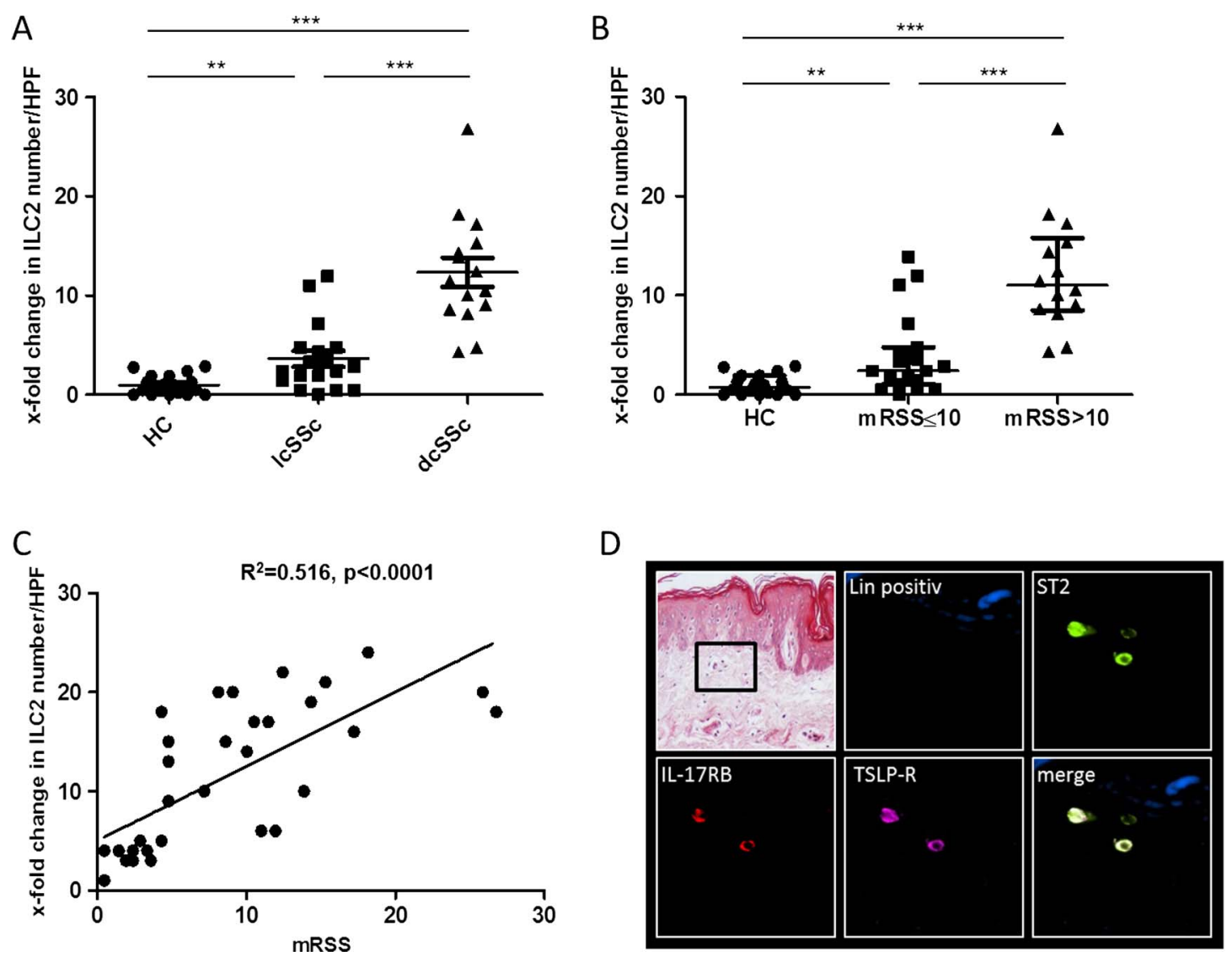

Figure 2 Type 2 innate lymphoid cell (ILC2) counts in the skin correlate with the fibrotic burden in patients with systemic sclerosis (SSC). (A) Numbers of ILC2s in skin sections of healthy controls (HC) and of patients with limited cutaneous SSc (IcSSc) and diffuse cutaneous SSC (dcSSc). (B) ILC2 counts in skin sections of HC and patients with SSC with an modified Rodnan skin score (mRSS) >10 compared with patients with SSC with an mRSS $\leq 10$. (C) Correlation between ILC2 numbers in the skin and the mRSS. Data are shown as median and IQR. (D) Representative images of H\&E and immunofluorescence staining for ILC2s in the skin of patients with SSC ( $n=5)$ using ILC2 activation markers TSLPR, interleukin (IL)-17RB and ST2 as positive markers and Lin (CD3, CD14, CD16, CD19, CD20 and CD56) as negative markers for ILC2s. HPF, high power field. *0.05 > p $\geq 0.01$; ${ }^{* *} 0.01>p \geq 0.001 ;{ }^{* * *} p<0.001$.

patients with extensive lung involvement assessed by CT scan (see online supplementary figure S5B). A comparison of circulating ILC2 frequencies between different cohorts of patients with rheumatoid arthritis, ${ }^{16}$ systemic lupus erythematosus ${ }^{17}$ and SSc demonstrated strong upregulation of ILC2s only in patients with SSc (see online supplementary figure S3A). Together, these data demonstrate that not only ILC2 numbers in tissue, but also in the blood are correlated with fibrotic manifestations in SSc.

\section{Multivariate analysis of clinical parameters and ILC2 numbers in patients with SSC}

Next, we calculated a multivariate linear regression model with three associated clinical parameters (lcSSc vs dcSSc; mRSS; the presence of pulmonary fibrosis) of our cross-sectional cohort to predict ILC2 numbers in a stepwise forward model and a stepwise backward model. Sex and age were also incorporated into the regression analysis as possible confounders. The best forward selection method led to a model consisting of the extent of skin fibrosis defined by dcSSc $(\beta=0.704 ; \mathrm{p}<0.001)$ and the presence of pulmonary fibrosis $(\beta=0.327 ; \mathrm{p}=0.005)$. The backward selection method led to the same model, thereby demonstrating the robustness of this data set. The correlation coefficient $(\mathrm{R}=0.885)$ results in a coefficient of determination $\left(\mathrm{R}^{2}\right)$ of 0.764 . Thus, the results from our cross-sectional cohort demonstrate that in patients with SSc as much as $76 \%$ of the variability of the ILC2 numbers can be explained by a combination of the extent of skin fibrosis and the presence of pulmonary fibrosis; however, as skin and lung fibrosis are dynamic variables over time, follow-up studies are necessary to evaluate ILC2s as predictors of fibrotic activity.

\section{DISCUSSION}

Herein, we provide first evidence for a role of ILC2s in the pathogenesis of rheumatic diseases by demonstrating increased ILC2 numbers in patients with SSc. Our data are indirectly supported by findings of increased ILC2 counts in animal models of fibrotic diseases such as $\mathrm{CCl}_{4}$-induced liver fibrosis and $\mathrm{S}$. mansoni-induced pulmonary fibrosis. ${ }^{56}$ In contrast to sophisticated studies in mice, data on ILC2s in humans are mainly limited to allergic diseases and largely focus on circulating ILC2s. ${ }^{18}$

Higher numbers of ILC2s in the skin as well as in the blood were both associated with more extensive skin fibrosis as shown by increased ILC2 numbers in patients with SSc with (i) dcSSc versus lcSSc, (ii) patients with high mRSS versus patients with low mRSS and (iii) a highly significant correlation of dermal and circulating ILC2 counts with the mRSS. In addition, patients with SSc with pulmonary fibrosis had significantly increased ILC2 counts in peripheral blood as well as in the skin (data not shown). The coexpression of skin-homing marker CLA and activation markers TSLPR and IL-17RB on skin resident in contrast to circulating ILC2s suggests that ILC2s are activated locally in the skin. ${ }^{15-17}$ The highly significant correlations 
Figure 3 The number of circulating type 2 innate lymphoid cells (ILC2s) is increased in patients with systemic sclerosis (SSc) and correlates with the extent of skin fibrosis. (A) ILC2 counts in the blood of patients with SSC and healthy controls (HC). (B) Numbers of ILC2 $s$ in the blood of patients with limited cutaneous SSc (ICSSC) and diffuse cutaneous SSc (dcSSc). (C) Circulating ILC2 counts of HC and patients with SSc with an modified Rodnan skin score (mRSS) $>10$ compared with patients with SSc with an $m R S S \leq 10$. (D) Number of circulating ILC2s in $\mathrm{HC}$ and patients with SSc with and without pulmonary fibrosis. All data are shown as median and IQR.
A

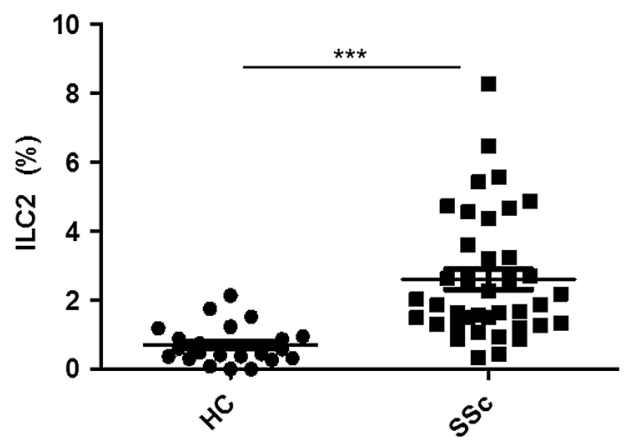

C

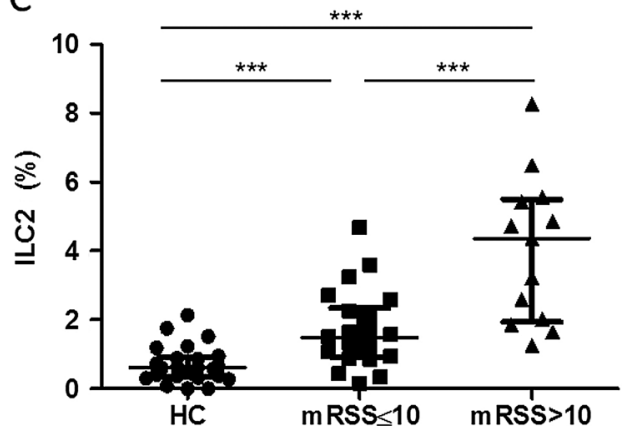

B
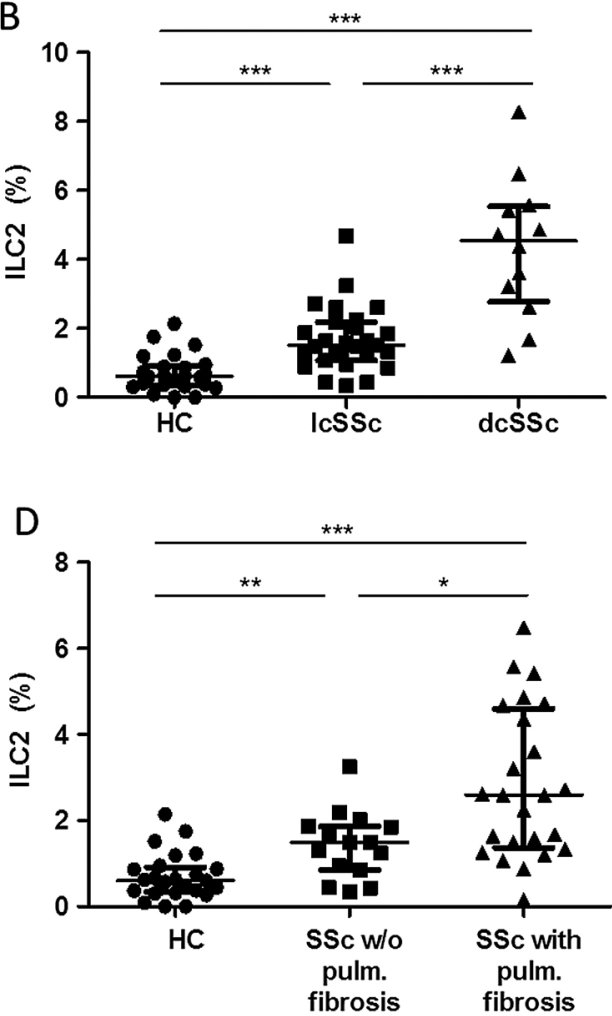

of ILC2 counts with the extent and severity of dermal and pulmonary fibrosis encourage follow-up studies to further evaluate the potential of ILC2s as biomarkers for fibrosis in patients with SSc. However, to serve as a biomarker, our findings need to be confirmed in independent, larger cohorts of patients with SSc with sufficient power to detect correlations with other, rarer fibrotic manifestations and the sensitivity of ILC2 counts to change over time needs to be further evaluated. The association of increased numbers of ILC2s in patients with SSc with fibrotic manifestations also warrants mechanistic studies to elucidate the molecular mechanisms by which ILC2s may promote fibrosis and to characterise the potential of targeting ILC2s as an antifibrotic approach in SSc.

Acknowledgements We thank Regina Kleinlein, Christina Weiss and Monica Pascual for excellent technical assistance.

Contributors Design of the study: TW, JHWD, AR. Acquisition of data: TW, SU, SW, AR. Interpretation of data: TW, ME, CD, CB, OD, GS, JHWD, AR. Manuscript preparation: TW, ME, CD, OD, GS, JHWD, AR. Provided patient samples: $C B, K G$, $O D, J H W D, A R$.

Funding Grants DI 1537/5-1, DI 1537/7-1, DI 1537/8-1, DI 1537/9-1, AK 144/ 1-1, RA 2506/3-1 and SCHE 1583/7-1 of the Deutsche Forschungsgesellschaft, Else Kröner-Fresenius-Stiftung 2014_A184, grants A57 and J40 of the Interdisciplinary Center for Clinical Research Erlangen (IZKF), the ELAN-Program of the University of Erlangen-Nuremberg and the Career Support Award of Medicine of the Ernst Jung Foundation.

Competing interests OD has consulted for, or has received research funding from, 4D Science, Actelion, Active Biotec, Bayer-Schering, Biogen, Biovitrium, BMS, Boehringer, EpiPharm, Ergonex, GSK, Inventiva, Medac, Novartis, Pfizer, Roche/ Genentech, Sanofi/Genzyme, Serodapharm, Sinoxa and United BioSource Corporation; JHWD has consultancy relationships and/or has received research funding from Actelion, BMS, Celgene, Bayer Pharma, Boehringer Ingelheim, JB Therapeutics, Sanofi-Aventis, Novartis, UCB, GSK, Array Biopharma and Active Biotech in the area of potential treatments of SSC and is stock owner of $4 D$ Science $\mathrm{GmbH}$.

Ethics approval University of Erlangen-Nuremberg.

Provenance and peer review Not commissioned; externally peer reviewed.

\section{REFERENCES}

1 Varga J, Abraham D. Systemic sclerosis: a prototypic multisystem fibrotic disorder. J Clin Invest 2007;117:557-67.

2 Gabrielli A, Avvedimento EV, Krieg T. Scleroderma. N Engl J Med 2009;360:1989-2003.

3 Beyer $C$, Schett $G$, Distler 0 , et al. Animal models of systemic sclerosis: prospects and limitations. Arthritis Rheum 2010;62:2831-44.

4 Wynn TA. Fibrotic disease and the $\mathrm{T}(\mathrm{H}) 1 / \mathrm{T}(\mathrm{H}) 2$ paradigm. Nat Rev Immunol 2004;4:583-94.

5 McHedlidze T, Waldner M, Zopf S, et al. Interleukin-33-dependent innate lymphoid cells mediate hepatic fibrosis. Immunity 2013;39:357-71.

6 Hams E, Armstrong ME, Barlow JL, et al. IL-25 and type 2 innate lymphoid cells induce pulmonary fibrosis. Proc Natl Acad Sci USA 2014;111:367-72.

7 Walker JA, Barlow JL, McKenzie ANJ. Innate lymphoid cells-how did we miss them? Nat Rev Immunol 2013;13:75-87.

8 Yokota Y, Mansouri A, Mori S, et al. Development of peripheral lymphoid organs and natural killer cells depends on the helix-loop-helix inhibitor Id2. Nature 1999;397:702-6.

9 Spits H, Cupedo T. Innate lymphoid cells: emerging insights in development, Lineage relationships, and function. Annu Rev Immunol 2012;30:647-75.

10 Hwang Y, McKenzie AJ. Innate lymphoid cells in immunity and disease. In: Katsikis PD, Schoenberger SP, Pulendran B, eds. Crossroads between Innate and adaptive immunity IV. New York: Springer, 2013:9-26.

11 Hoyler T, Klose Christoph SN, Souabni A, et al. The transcription factor GATA-3 controls cell fate and maintenance of type 2 innate lymphoid cells. Immunity 2012;37:634-48.

12 Yang Q, Saenz SA, Zlotoff DA, et al. Cutting edge: natural helper cells derive from lymphoid progenitors. J Immunol 2011;187:5505-9.

13 Spooner CJ, Lesch J, Yan D, et al. Specification of type 2 innate lymphocytes by the transcriptional determinant Gfi1. Nat Immunol 2013;14:1229-36.

14 Gorski SA, Hahn YS, Braciale TJ. Group 2 innate lymphoid cell production of IL-5 is regulated by NKT cells during influenza virus infection. PLoS Pathog 2013;9:e1003615.

15 Salimi M, Barlow JL, Saunders SP, et al. A role for IL-25 and IL-33-driven type-2 innate lymphoid cells in atopic dermatitis. J Exp Med 2013;210:2939-50.

16 Teunissen MB, Munneke JM, Bernink JH, et al. Composition of innate lymphoid cell subsets in the human skin: enrichment of NCR+ILC3 in lesional skin and blood of psoriasis patients. J Invest Dermatol 2014;134:2351-60.

17 Eberl G, Colonna M, Di Santo JP, et al. Innate lymphoid cells: a new paradigm in immunology. Science 2015:348:aaa6566.

18 Bartemes KR, Kephart GM, Fox SJ, et al. Enhanced innate type 2 immune response in peripheral blood from patients with asthma. J Allergy Clin Immunol 2014;134:671-8.e4. 


\section{A RD Type 2 innate lymphoid cell counts are increased in patients with systemic sclerosis and correlate with the extent of fibrosis}

Thomas Wohlfahrt, Svetlana Usherenko, Matthias Englbrecht, Clara

Dees, Stefanie Weber, Christian Beyer, Kolja Gelse, Oliver Distler, Georg Schett, Jörg H W Distler and Andreas Ramming

Ann Rheum Dis2016 75: 623-626 originally published online September 2, 2015

doi: 10.1136/annrheumdis-2015-207388

Updated information and services can be found at:

http://ard.bmj.com/content/75/3/623

These include:

Supplementary Material

References

Email alerting service
Supplementary material can be found at:

http://ard.bmj.com/content/suppl/2015/09/02/annrheumdis-2015-2073 88.DC1

This article cites 17 articles, 4 of which you can access for free at: http://ard.bmj.com/content/75/3/623\#ref-list-1

Receive free email alerts when new articles cite this article. Sign up in the box at the top right corner of the online article.

Collections

Articles on similar topics can be found in the following collections

\section{Notes}

To request permissions go to:

http://group.bmj.com/group/rights-licensing/permissions

To order reprints go to:

http://journals.bmj.com/cgi/reprintform

To subscribe to BMJ go to:

http://group.bmj.com/subscribe/ 